\title{
The Research of The 35KV optimization design and stable operation of technical for wind farm
}

\author{
Jingjing Zheng, Yong Yang, Jianhua Zhang, Peidong Du, Yongjun Han \\ Gansu Electric Power Research Institute, Gansu, China \\ jingjing9@163.com
}

Keywords: harmonic, ungrounded system, ferromagnetic resonance, identifying the line in a single phase to ground.

\begin{abstract}
Large-scale accident which wind turbines are off the grid has happened in the base of Jiuquan wind farm. The majority of accidents are closely related to unreasonable operation technology and unreasonable run manner of $35 \mathrm{kV}$ system of wind farm, unreasonable selection of equipment.Study the wiring structure of the $35 \mathrm{kV}$ system of the wind power, the actual operation of the wind power, the restrict of technical means, reference current proven advanced technology, propose optimization from system design, equipment selection and run manner etc. Operation reliability of $35 \mathrm{kV}$ wind power system has been great increased.
\end{abstract}

\section{Introduction}

Since the $19 \times 200 \mathrm{MW}$ wind farm in Jiuquan wind base was in operation, $35 \mathrm{kv}$ stable heads has been out of order for many times.Because $35 \mathrm{kv}$ wind farm system is ungrounded, there is no protection from single-phase grounded trip, so the ground branch can not be cut off on time. The faults get worse, in result, large-scale wind turbine in the whole Jiuquan area was off the grid, besides, the whole Ganshu grid was widely and badly influenced.

The accident of $35 \mathrm{kv}$ system of $200 \mathrm{MW}$ wind farm in one wind base in Jiuquan. On April 17 th, 2011, in one wind farm in Jiuquan, the $35 \mathrm{kV}$ distribution room D busbar voltage transformer three-phase grounding fault occurred, causing fluctuation of system voltage and the wind turbine was wholy off the of grid.Moreover, 700 fans including all the wind turbines of this wind farm are off the grid, losing the output as $928 \mathrm{MW}$.

Firstly, the cable heads of 35C2 feeder does not work, leading single-phase grounded. Then 35D2 feeder is interphase short circuited, the fault shown as follows, in figure 1. From the single-phase grounding to cutting off faults, ferro resonance of $35 \mathrm{kv}$ busbar potential transformer was caused, and huge inrush current appeared,the current of voltage transformer increases tenfold one time.saturation voltage of voltage transformer was caused At length,the insulator of $35 \mathrm{kv}$ busbar voltage transformer was damaged, which causes the bus was three-phase short circuited,the voltage transformer was burn out.The recording wave of this accident shown as figure 2 and figure 3 .

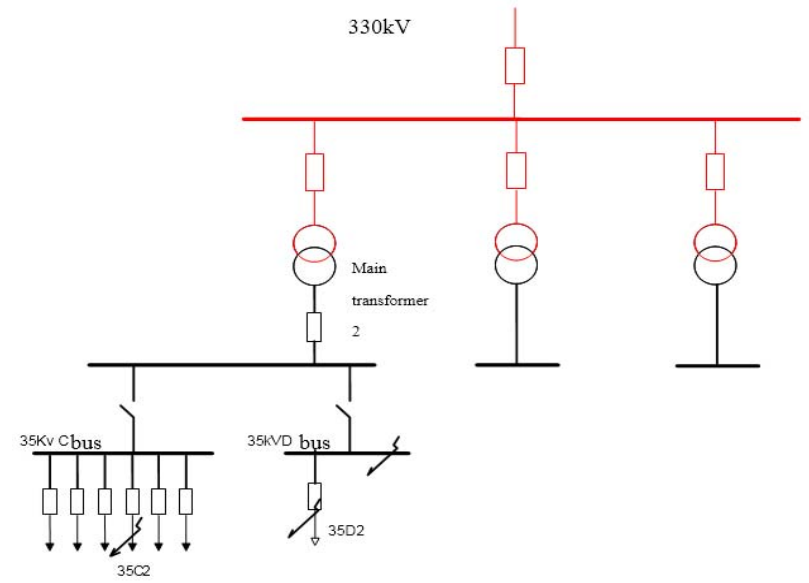

Fig. 1 Wind farm booster station $35 \mathrm{KV}$ D bus tripping fault 


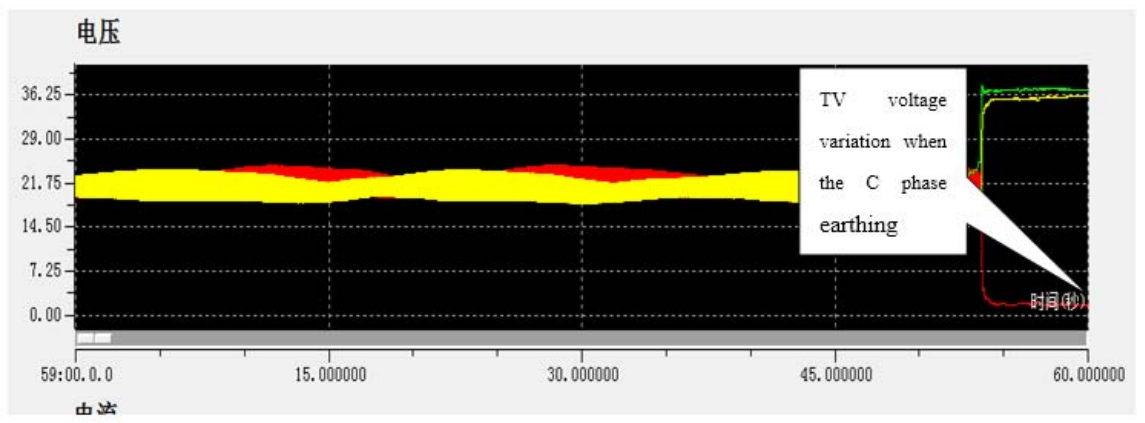

Fig. $235 \mathrm{kV}$ D Bus

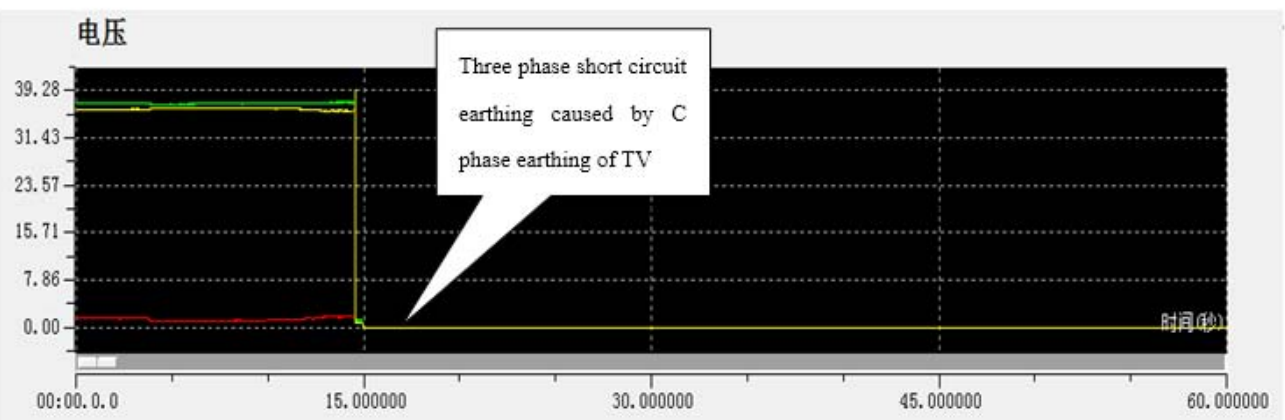

Fig. $335 \mathrm{kV}$ D bus three phase earthing short circuit voltage curve(05:00:14)

The problem revealed by accident. In Some wind farms , TCR typed (thyristor controlled reactor) SVC beside $35 \mathrm{kv}$ system in some wind farms. During function testing, it is found that when TCR is in operation, the distribution flow of harmonic voltage is relatively large, some data has exceeded standards. Long time affected by harmonic, it would cause damage to the insulators of whole $35 \mathrm{kv}$ system, especially to many $35 \mathrm{kv}$ cable heads in the wind farm.

(2) In $330 \mathrm{kV}$ booster station, there exists shortcomings in $35 \mathrm{kV}$ system design. Under the present operation mode, when $35 \mathrm{kv}$ system has single-phase grounding or other faults, the ferromagnetic resonance of voltage transformer was easily caused.

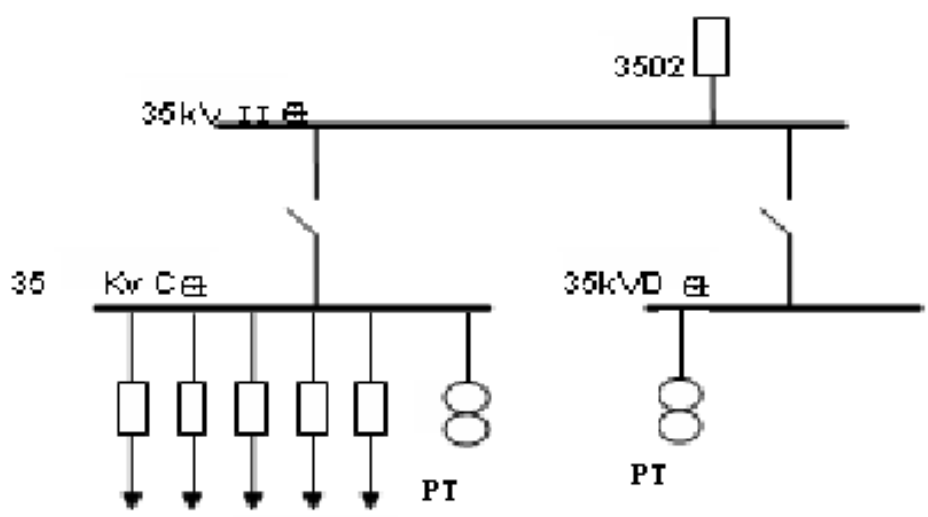

Fig. 4 200MW wind farm typical 35kV

Shown as figure 4 , the $35 \mathrm{kv}$ system of every single wind system divides into two buses (parallelly operating through isolator), each bus is equipped with one electromagnetic voltage transformer, which means operating two sets of voltage transformer on each bus. According to the analysis of principles of ferromagnetic resonance. This sort of joint design is more apt to induce ferromagnetic resonance of electromagnetic voltage transformer during the transient process.

There exists problems in design and equipment selection, the ungrounded system of $35 \mathrm{kV}$ system lacks the protection for single-phase grounding. If single-phase grounded, but the fault point can not be cut off immediately, the $35 \mathrm{kV}$ system healthy voltage would lifts.If it cann not be dealt with immediately, the $35 \mathrm{kv}$ system, especially the relatively weak cable heads would bear the 
burden of long time overvoltage.The accident would be more easily worsen into interphase or three-phase short-circuit.

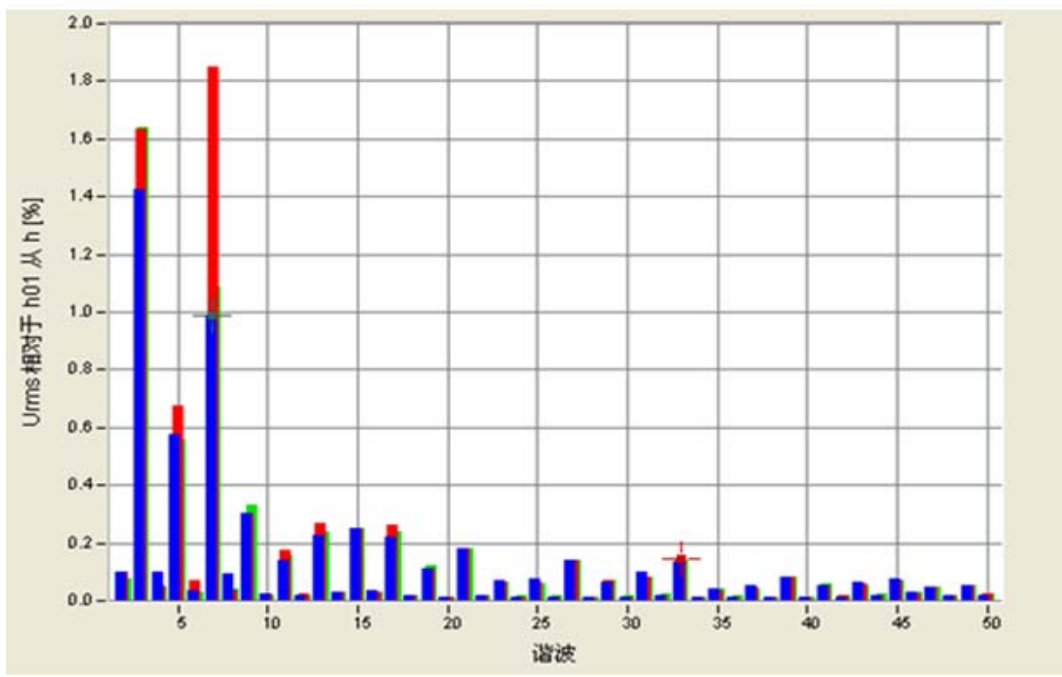

Fig. 5 TCR typed SVC operation $35 \mathrm{kV}$ bus harmonic voltage relative to the base wave spectrum

\section{Analysis on faults of 35KV system in 200MW wind farm and proposal}

Influence of TCR typed SVC power quality and proposals. TCR based SVC, which is relatively mature in technique, has many strong points such as immediately restraining voltage( with response time less than $30 \mathrm{~ms}$ ), saving energy, smoothly controlling reactive load permitting fluctuation, stable load. TCR can contuniously regulate output power, but its wave is zigzag-shaped, a huge harmonic source.Its harmonic current spectrum is shown as follows:

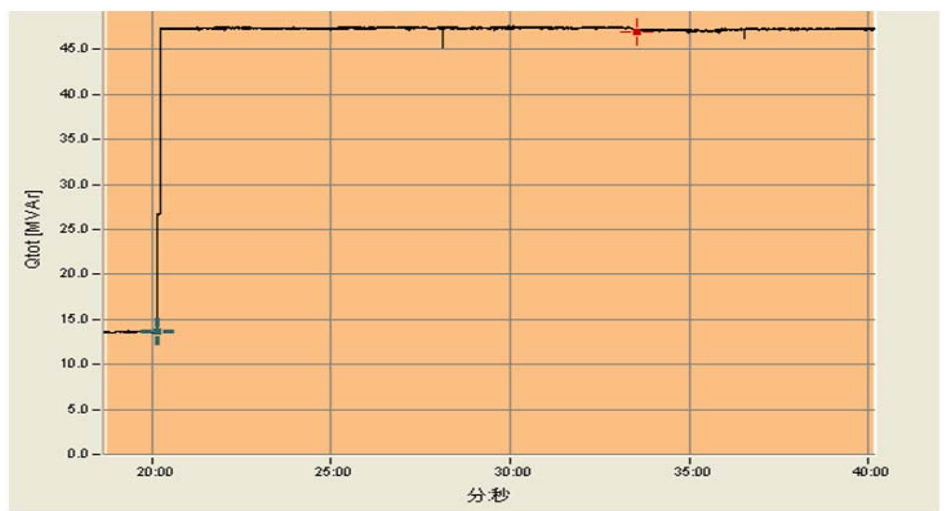

Fig. 6 TCR typed SVC operation $35 \mathrm{kV}$ bus harmonic current absolution the absolute value spectrum

The rationale of TCR demands higher power quality operating under high load than that under low load reactive output variation curve of TCR and absolute variation curve of three harmonic current in the same period are shown as figure7, figure8.

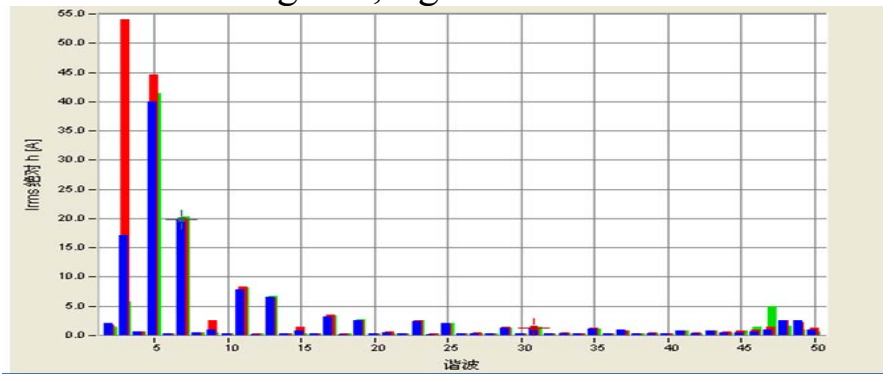

Fig. 7 TCR reactive outgoing power variation curve 


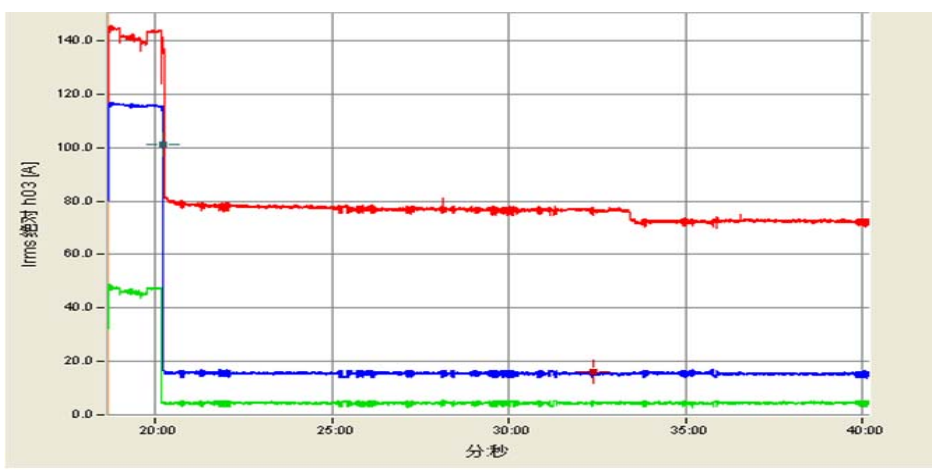

Fig. 8 TCR reactive power outgoing variation 3 times harmonic current absolution variation curve

It can be found that TCR reactive output power response rather fast from figur7, which shows its loading steps from 14MVar to 48MVa. When the reactive output power steps, three harmonic current vary dramatically, shown as figure8. The general current distortion rate was improved. The variation trend accord with working characteristics of TCR, the stronger reactive output power, the conduction angle opens larger, the current distortion occurs less.

In order to improve the power quality of TCR operation, TCR typed SVC equipped with filter capacitor banks are more common. In operation, in order to regulate bus voltage more effectively, switching filter capacitor frequently, which leads lack of restraining harmonic voltage.In Long-time operation, harmonic would cause irretrievable damage to $35 \mathrm{kv}$ system, especially to many cable heads of wind farm.

Therefore,TCR branch should be manipulated under high reactive output aiming to lessen harmonic. Meanwhile,the wind farm should regularize the operating mode of SVC and match filter capacitor banks with SVC, which guarantee the inhibited effect of harmonic voltage current.

The design,type section, and operating mode of optimizing $35 \mathrm{kv}$ system voltage transformer $35 \mathrm{kV}$ system has voltage transformer on its bus, Usually, its winding is star-shaped, neutral directly grounded.So when the system operates(for example,neutral indirectly grounded system closing non-synchronously,or single-phase earth fault, voltage transformer the magnetizing impedance of voltage transformer would formulate ground capacitance nonlinear resonance loop with ground capacitance of system, and cause ferro resonance overvoltage Moreover, due to the difference between circuit parameters and external shooting condition, which may cause frequency division, power frequency or high frequency ferro resonance overvoltage.According to operating experience and statistical data of power system shows that statistical data caused by electromagnetic voltage transformer is kind of internal overvoltage in the neutral ungrounded system, which is most common to see and cause accidents internal overvoltage, The safety and stable operation of power system would be severely damaged. So enough attention must be paid.

It is advised that anti-harmonic voltage transformer or neutral point single-phase potential transformer should be adopted. With these two measurements, When the line is single phase grounded, each phase the voltage of voltage transformer should be maintained as normal-voltage but unsaturated, which enhances zero sequence excitation specialty, of voltage transformer, and low down its primary current.Meanwhile,it ensures that grounding line selection device sensitive to ratio and phase. $35 \mathrm{kV}$ bus are equipped with two sets of electromagnetic voltage transformer and formulate non-linear harmonic circuit with distributed capacitance of many sets of feeder cables. Ferro resonance is easily induced when the system is single grounded.Therefore, its design is advised to change, with extra voltage parallel arrangement of bus. When one voltage transformer applied, through parallel arrangement, all TV voltage come from automatic operated voltage transformer. Thus LC parameter of system can be changed and harmonic resonance.

Perfect the protect function of line selection device for low current of ungrounded system. Traditional line selection device for low current,generally adopt warning due to its low line selection rate. Such rational is referred in the wind farm design selection. 
The original design does not take the protect function of $35 \mathrm{kv}$ system single-phase grounded fault.If the fault point can not be cut off immediately when single-phase grounded, $35 \mathrm{kV}$ non-faulted voltage phase lifts and operates would worsen the fault as interphase short circuit or three-phase short-circuit。So, it demands change in wind farm, line selection device with the function of two tripping for low current. When single-phase grounded, line selection device for low current was selected, the chosen branch would be cut off immediately.In the same time, if it is correctly selected and cut off, the fault point disappear and line selection device returns, the healthy branch can operate normally, which ensures that wind farm loss.the turbine generator output of merely one $35 \mathrm{kv}$ feeder.If it is wrongly selected,the fault point still exists after first round trip, at second round trip, rebound $35 \mathrm{kV}$ side switch of main wind farm. Separate the whole wind farm from power grid to guarantee that the grid safety affected by single wind farm fault. Meanwhile, in order to avoid the risk of wrongly cut off fault due to low accuracy of line selection device for low current. The line selection should be of high accuracy and advanced principles.

Mode design through resistance or arc suppression coil grounding adopted by $35 \mathrm{kv}$ system. Considering the demand of quickly remove the wind farm feeder when single-phase faulted.The feeder can adopt the mode design through resistance or arc suppression coil grounding and abandon the original ungrounded or Peterson coil earthing mode. When the feeder system is single-phase earthing to ground, it can be immediately cut off through relevant protection.Moreover, the adaptivity of operating unit voltage should also be accounted. When feeder system grounded through arc suppression coil single-phase grounded, the reliable line sections should be chosen and cut off quickly.Feeder protection point flow rate certification of feeder protection point should bare sensitivity towards end fault, the busbar differential protection should also be equipped on $35 \mathrm{kv}$ bus.

\section{Conclusion}

Jiuquan wind farm base develops rapidly, the present installation capacity has almost amounted to $5000 \mathrm{MW}$. which tops the world.Many large scale load rejection caused by $35 \mathrm{kv}$ system faults here has posed huge threat to the power grid, and cause enormous economic losses.

Analysis of this fault proves that $35 \mathrm{kv}$ system of wind farm in large scale wind power base is the weak link in operation.Because the wind turbine in Jiuquan has special mode of large-scale cluster network.Confronting such special mode, $35 \mathrm{kv}$ system has no mature design, selection type and operation experience.By means of optimizing $35 \mathrm{kv}$ system in its design, type selection and operation technology, the level of stable and safe operation of Jiuquan wind base can be improved with large amplitude.

\section{Reference}

[1] Ma Hong fei. Xu Dian guo. Miao Li jie. Scheme comparison \& analysis of four types VSCF wind power generation system[J]. Electrotechnical Jounral. 2000(10): 1-4(in Chinese)

[2] Dai Hui zhu. Wang Wei sheng, Chi Yong ning. Recent wind power integration study in China[J]. Power System Technology, 2007. (20): 16-23(in Chinese).

[3] Xi Xiao. Li Haiyan. Kong Qin gd on g. Influence of connecting and discormecting wind farm with regional power network on voltage of the later[J]. Power System Technology. 2008. 32(10): 58-62(in Chinese)

[4] Li Yu, Fan Gaofeng. Li Ding, et al. The impact of Da ban cheng wind farm integration on power quality of Xinjiang power grid[J]. Power System Technology. 2007. 31(6): 88-92(in Chinese)

[5] Lei Ya zhou, Wang Wei sheng. Yin Yong hua. et al. Analysis of wind power value to power system operation[J]. Power System Technology. 2002. 26(5): 10-14(in Chinese) 
[6] 2008. 32(14): 15-18.Wen Yu ling, Chao Qin. Tuerxun Yibulayin . Impact of interconnected wind farm on power system protection[J].Power System Technology,2008, 32(14): 15-18(in Chinese)

[7]Zhou Lixia. gin Zhongdong. Zheng Li.Research on Principle of PT Resonance in Distribution Po wer System and Its Suppression[J].Transactions of China Electrotechnical Society. 2007, 22 (5): 153-158(in Chinese).

[8] State grid corporation grid 18 major anti-accident measures (revised edition)[S]. 